\title{
Discourse Misunderstanding from a Perspective of Relevance Theory
}

\author{
Yu Zhang \\ The Tourism College of Changchun University \\ Changchun, China 130607
}

\author{
Junyan $\mathrm{Li}^{*}$ \\ Changchun University of Finance and Economics \\ Changchun, China 130122 \\ *Corresponding Author
}

\begin{abstract}
Nowadays, discourse misunderstanding appears frequently in daily life. Why discourse misunderstanding is formed? It is a kind of obstacles that hinder communication. This thesis explains the problems from cognitive language, an angle of Relevance Theory in order to achieve a fluent communication.
\end{abstract}

Keywords-discourse misunderstanding; Relevance Theory; communication

\section{INTRODUCTION}

French and British scholars, Sperber and Wilson have produced Relevance Theory from a perspective of cognition in 1986. [1] They consider language communication is kind of ostensive-inferential process, which are the two aspects of communication process. For speaker, communication is an ostensive process. It refers to an action that speakers ostensively express his or her attention. The purpose of this action asks the hearer's manifest. Therefore, this process can achieve mutual manifest. While for hearer, communication is a process of inference process. The hearer infers the speaker's intention through ostensive information correlated with context in order to come to the best contextual effectiveness. This thesis focus on the problems that may happen in the points on ostensive information, context and inference process and try to find the reasons that cause discourse misunderstanding.

\section{REASONS FOR DISCOURSE MISUNDERSTANDING FROM A PERSPECTIVE OF RELEVANCE THEORY}

He Ziran has mentioned communication is a cognitive process. The reason why the two sides collocate with themselves well is mainly due to the best cognitive mold relevance. [2]Relevance is the basic factor restricting human's communication. From a math perspective, it is a given. Relevance can be defined as a means rather than a final purpose for ostensive action. Relevance Theory considers context as a serious of hypotheses in our mind in order to interpret language correctly through interactive process. the context may consists of specific situation, language context, knowledge and characteristics of social mentality.[3] Cognitive context is active and also a variable from math perspective .so , as to the information about Relevance Theory, It depends on the relevance between information and specific context. Therefore, through analysis above, there are three key aspects that can lead to discourse misunderstanding in communication. They are ostensive information, specific context and inference process.

\section{A. Insufficiency for Ostensive Information}

According to Relevance Theory, the speaker says sentence which contains ostensive characteristics. That is to say, the sentence is unambiguous, definite. So the hearer will has direction and objective for his or her inference and judge exactly the speaker's intention. While, if the speaker's words contains insufficient information that he or she wants to express. The hearer will not construct relevant mold. So discourse misunderstanding will appear. It may result into obstacles in communication.

There was a conversation between a mother and her daughter about. Her daughter is very fat but single. (A represents mother, B represents daughter)

A: Honey, Have you dating with boy?

B: No, I am trying to find my Mr. Right. But I can't make it.

A: while, (mother moves into kitchen) what do you want to eat tonight?

B: I want a piece of that beefcake.

A: What? Beefcake? You must keep your body slim.

$\mathrm{B}$ : Oh, Mom (the daughter is so angry)

From this example, there appears discourse misunderstanding. The word "beefcake" is the root causing misunderstanding. Beefcake is a very vogue word which describe the boy has a strong built and pretty sexy. Mother has never heard "beefcake" but she has ever heard cream cake, birthday cake, any kinds of cakes. In her mind, every kind of cake contains fat. It will destroy her daughter's beauty. So mother's answer lead to misunderstanding. In the mother's dictionary, she knows some kinds of cakes and the information about cake is certainly existing .But the mother's cognitive degree is fewer than her daughter. In this situation, when the ostensive information is not enough, it will lead to obstacles in communication. Actually, her daughter is not purposely saying like that. As her mother went the kitchen, she didn't hear her mother's voice. From the perspective of Relevance theory, this discourse misunderstanding is due to 
the insufficient ostensive information resulting into bad collocation between ostensive information and the mother's specific context. Therefore, discourse misunderstanding will appear.

\section{B. Ambiguity of Ostensive Information}

Language has arbitrariness. From an angle of language itself, language symbols are restricted, while objective world is unlimited. Ambiguous phenomenon is unavoidable by using restricted symbols to express unlimited matters. Subjectively, when human being know world and divide it into categories, they must obey cognitive economical principle and language economical principle. This will be sure to result into ambiguous meaning on category and lexicon. Objectively, the ambiguity of human being's thinking and uncertainty of context will inevitably lead to the ambiguity of choosing language.

From the perspective of Relevance Theory, ostensive information plays a very important role. If the transmitted information is clear to understand, an appropriate relevance will be achieved. While the words transmitted is ambiguous resulting into a failing communication.

There is a couple and a salesman who sells sweets. In the couple's house, a salesman tried to sell his sweets (A: wife B: husband C: salesman)

C: What taste do you like best? I have mints, toffee, fruit candies, gummy jelly. (the salesman introduced every kind of sweets with patience)

B: Who are you talking with? (the husband just walked from bedroom)

\section{A: A sweet salesman. \\ C: (the salesman feels surprised and spoke nothing.)}

The piece of conversation also exists discourse misunderstanding, It goes fluently at the beginning. However, the word "sweet" has ambiguous meaning. It is can be defined as an adjective, "cute" and also considered as a noun, "candy". According to the two different explanations, it must be different reflections. Apparently, the salesman considered "sweet" as "candy" rather than "cute". That is to say, when the speaker spoke the ostensive information that is ambiguous, in this situation, the hearer will certainly construct relevance. However, it is a failing relevance. So he may think the wife looked down upon him and be angry. Discourse misunderstanding will appear.

There is another piece of conversation to further explain ambiguity of ostensive information causing discourse misunderstanding. It is a conversation between a boss and his employee discussing another employee, John. (A: boss B: employee)

\section{A: What has John been busy doing recently?}

B: I met him yesterday. He decided to work hard when he was promoted to be manager of the sales department.

A: Oh, he is so lazy. (the boss is frowning)
In this conversation, the Boss wanted to know something about John in order to decide to promote him as a manager whether or not. However, the sentence spoke by the employee is ambiguous. One explanation is that John would work hard after he was promoted. The other explanation is that John had always been working hard before he was promoted to be manager. The ostensive information gave the boss the first explanation rather than the second one. The boss would consider John is a lazy person. So the boss would construct the inappropriate relevance according to the specific context. Therefore, this thesis can consider ambiguity of ostensive information may make failing relevance and is another reason that causes discourse misunderstanding.

\section{Lack in Context}

Relevance Theory focuses on ostensive information, inference and context. Context is decisive and necessary and plays an essential role in inference process. People communicate with themselves, the speaker's purpose of communication is to achieve manifest from the hearer. So the speaker and hearer can come to mutual manifest. However, when the hearer's context completely doesn't include the speaker's context, that is to say the hearer will lack in context. It will result into obstacles in communication.

Here is another conversation happened in a restaurant. A Chinese people ordered a dish in an American restaurant. (A: customer B: waiter)

A: Would you please serve the Bird's nest in a clear soup?

B: No, I can't.

A: why not?

\section{B: Are you kidding?}

From the example above, there is another discourse communication. In Chinese context, that is to say, In Chinese culture, there certainly has a dish called "Bird's nest in a clear soup". The term translated into Chinese is named “清汤 燕窝”.While in western context, or western culture, there certainly hasn't a dish like that. That is to say, when speaker spoke the word “清汤燕窝”. It will not get any manifest from the hearer. That is due to lack in this dish in western people's dictionary. So when the western people heard the strange name, the two sides will not get mutual manifest. The hearer will consider the customer coming here is to make trouble and be angry. It will bring about barrier in communication. Therefore, lack in context is another reason resulting into discourse misunderstanding.

\section{Sudden Change for Context}

Language explanation is the process in which the hearer will find the best relevance. If the hearer can't find the appropriate relevance in the small context, he or she should try to find the relevance in a large context. So the speaker and hearer will communicate with themselves fluently. While, if the speaker can't find the correct context for his or her relevance. The conversation will be failed. 
There is another conversation between two classmates. They came across in a library. (A: Vivien B: Candy)

Vivien: How time flies! How about your preparation for our final exam?

Candy: The weather has been quite delightful this summer, hasn't it?

Vivien: What? Why do you say like that? Have you prepared for your final exam?

\section{Candy: (She is going on reading and keeping silent)}

Apparently, there is another kind of discourse misunderstanding. B didn't want to talk about the preparation of her final exam. Maybe she hadn't prepared for the final exam or she had prepared but not already. So she changed the topic that is to say, she changed a large context. However, A can't understand why B changed the other topic and she can't find an appropriate context for her relevance. Finally, It will bring about a kind of misunderstanding. Through the analysis of Candy's words, it contains a sort of potential relevance. B purposely changed topic and the purpose is to change a relaxed topic rather than a heavy one. Regretfully, A can't understand B's real intention. It inevitably appeared discourse misunderstanding.

There happened another conversation between a man and a female. The man wants to propose marriage to the girl. (A: boy B: girl)

A: You're the person of my dreams. I think I might be falling in love with you. Will you marry me?

B: Oh, have you ever heard a very romantic song, called "I will always love you"

A: Have you go on listening to my speaking? I need your direct answer.

From the example above, there appears a specific context that is a man wants to propose marriage to a female. However, the relevant context is suddenly changed. The man can't construct appropriate relevance in a hurry. So there produced obstruction in communication. Actually, the female suddenly changed the topic. This action contains a kind of implicature. The new context has a certain relationship with the male's context. Regretfully, the male will not achieve manifest. So discourse misunderstanding will appear and the conversation will get into an embarrassed situation. Therefore, sudden change of context is another reason that will cause discourse misunderstanding.

\section{E. Mistake in Inference Process}

Relevance theory attaches importance on ostensive and inferential process. The hearer receives the ostensive information and infers the speaker's meaning by specific context. While, this kind of inference may come to different results according to the hearer's knowledge, state of mind, specific situation, etc. All outside subjective and objective factors may influence the hearer inferential process. So, when there comes out a piece of ostensive information from the speaker, maybe there is only one answer that is reasonable. It is due to the correct inference process and appropriate relevance. Other inferences will result into discourse misunderstanding.

There is another conversation between a professor and his students. It was a hot afternoon. The electric fans broke down in the classroom. A professor is giving a lecture to his students. (A: teacher B: student $1 \mathrm{C}$ : student $2 \mathrm{D}$ : student $3 \mathrm{E}$ : student4)

A: What a hot day! (the professor put up his head looking at the fans)

B: Oh, yes! Today's temperature is 40 degree centigrade by weather forecast.

\section{$\mathrm{C}$ : If only there is an air conditioner!}

D: You are so painstaking.

E: Sorry, professor, a mechanic in water-electric department said he would repair the fans tomorrow.

Apparently, there appeared different answers. When B heard the professor's ostensive information, he certainly constructed a kind of relevance between the information and context. He made his own inference according to his own experience that he had known today's temperature by weather forecast. So he directly spoke out and can't understand the professor's real intention. When $\mathrm{C}$ heard professor's ostensive information that is so hot today, according to the specific context and his knowledge, what is his first impression is air conditioner is cooler than fans. So he constructed a wrong relevance and his inference is also wrong. When D heard the professor's words, he also constructed certain relevance and he inferred through his own state of mind. Although it is hot today, the professor still gave a lecture. He would think that the professor is a very hardworking, even painstaking person. This kind of relevance is failing and also an inappropriate inference. Although the three inferential processes are different, they all cause discourse misunderstanding. While student D is an opposite example. He made a correct inference. He judged the relevance by the professor's non-verbal language that is put up his head looking at the fans. Therefore, mistakes in inference process can also cause barrier in communication.

\section{CONCLUSION}

The thesis has concluded five reasons that can result into discourse misunderstanding. Among them, some are purposely spoke out by speakers, while others are not. The process of language communication is the psychological process that contains providing information, contextual participation, looking for relevance, inferring intention and finishing communication. Although Relevance Theory can't absolutely make exact communication between the hearer and the speaker, It has theoretical and practical function for avoiding discourse misunderstanding and words expression and explanation. Therefore, if the speaker and the hearer can correctly comprehend and manipulate Relevance Theory, simultaneously, giving other people more tolerance and care, it must be successfully avoiding certain discourse misunderstanding. 


\section{REFERENCES}

[1] Sperber Dan. \& Deirdre Wilson. Relevance: Communication and Cognition[M].Oxford: Blackwell,1986.

[2] Barbara.A.Spellman. Current Directions in Cognitive Science.[M] Beijing Normal University Publishing Group.2007.

[3] He Ziran. Grice's Pragmatic Doctrine and Relevance Theory[J]. Foreign Language Teaching and Research.1995,(4).

[4] He Zhaoxiong. A New Introduction to Pragmatics.[M].Shanghai::Shanghai Foreign Language Education Press, 2000.

[5] Sun Ya. Introduction to Pragmatics and Cognition [M] .Peking University Press. 2008. 